\title{
Ingeniería Física, un Título Profesional Universitario de la Física Aplicada para el Ámbito Productivo
}

\author{
Claudio A. Faúndez y Joaquín F. Díaz-Valdés \\ Universidad de Concepción, Facultad de Ciencias Físicas y Matemáticas, Departamento de Física, \\ Casilla 160-C, Concepción-Chile (e-mail: claudiofaundez@udec.cl; jdiaz@udec.cl)
}

Recibido Ene. 15, 2011; Aceptado Feb. 17, 2011; Versión final recibida Abr. 01, 2011

\begin{abstract}
Resumen
En este trabajo se describe la especialidad de Ingeniería Física como una de las ramas de la carrera Licenciatura en Ciencias Físicas. Dicha especialidad fue propuesta por un grupo de académicos del Departamento de Física de la Universidad de Concepción en Chile a comienzos del año 2000 y fue aceptada por las autoridades universitarias como una especialidad formal el año 2003. La principal idea que motivó a este grupo de académicos a crear esta especialidad, tema ya expuesto por la Comisión Económica para América Latina (CEPAL), fue generar un fuerte vínculo universidad-empresa el cual no se logra solamente con el aumento del número de científicos. La buena acogida que han tenido nuestros alumnos en sus prácticas profesionales muestra que el sector productivo ha sido muy receptivo de los estudiantes de esta especialidad augurando así un buen campo laboral en el futuro.
\end{abstract}

Palabras clave: ingeniería física, planes de estudio, física aplicada, propuesta docente

\section{Engineering Physics, a Professional Title of Applied Physics for the Productive Area}

\begin{abstract}
This paper describes the major of Engineering Physics as one of the applied branches of the career Bachelor in Physical Sciences. This major was proposed by a group of professors of the Physics Department of the University of Concepción in Chile at the beginning of the year 2000. This new career was accepted by the university authorities as a formal profession in 2003 . The main idea that motivated this group of professors for creating this major, topic already discussed by the Economic Commission for Latin America (ECLA), was to generate a strong link universityindustry. The good reception that the students had have in their industrial training programs show that the productive sector has been very receptive of the students of engineering physics, indicating that they will have good working opportunities in the future .
\end{abstract}

Keywords: physics engineering, plans of studies, applied physics, academic program 


\section{INTRODUCCIÓN}

La mayoría de los países latinoamericanos han realizado durante los últimos 17 años grandes esfuerzos para disminuir su dependencia económica de las exportaciones de materias primas, tratando de incorporar valor agregado a este tipo productos, sin lograr a la fecha los frutos deseados. Desde la perspectiva empresarial es conocido que para seguir creciendo de manera sostenible se requiere desarrollar capacidades de innovación permanente y estrategias bien definidas. Además, estos países han comprendido que recursos humanos y desarrollo son dos temas muy vinculados entre sí (OMPI-CEPAL, 2003, CEPAL 2010, Moya-Angeler, 2010). De lo anteriormente señalado es clara la necesidad de contar con profesionales con mayor capacidad de innovar y no solamente creadores de nuevas ideas o productos desvinculados de las necesidades del mercado, los cuales serían meramente inventores.

Por otro lado, ya existía experiencia previa de fuertes vínculos entre universidades y desarrollo tecnológico e industrial en los Estados Unidos de Norteamérica y la Universidad de Lehigh, formalizado en 1924 (Yates, 1992) y en Brasil en la década los ochenta cuando se crea INMETRO. Tanto autoridades como organizaciones gubernamentales, no gubernamentales, empresariales y académicas, han tomado conciencia de la gran cantidad de recursos humanos que se encuentran al interior de las universidades, donde hay una vasta trayectoria en la generación de conocimiento, ya sea basado en nuevas teorías o evidencia experimental, y de la necesidad de generar un fuerte vínculo universidad-empresa (OMPI-CEPAL, 2003).

Dentro del mundo académico, específicamente los físicos, se ha reconocido una gran oportunidad de desarrollo y diversificación del campo laboral, como por ejemplo en el área biomédica (Castrillón et al. 2009) que se hacen evidentes con la creciente necesidad de establecer una conexión entre la creación de conocimiento y la aplicación de éste con un criterio emprendedor (López et al., 2007;), es decir, desarrollar un vínculo sinérgico entre la física teórica y experimental con los procesos productivos, insertando así al físico al medio en el cual habita. En la actualidad se piensa que ese vínculo entre el mundo académico y empresarial, lo podría conformar en gran medida la física aplicada, si este concepto es plasmado como una especialidad dentro de una carrera universitaria en ciencias físicas. De esta manera, las posibilidades de desarrollo profesional de los estudiantes de física y su contribución al ámbito productivo, una vez titulados, serían de gran relevancia (Flores et al. 1981).

Por las razones expuestas, investigadores del área han decidido explotar este gran nicho que se ha presentado con mucha fuerza en la actualidad, y así poder utilizar la enorme capacidad para crear e innovar que ofrecen las ciencias físicas. Como muestra de ésta creciente necesidad en el mercado y la búsqueda de las soluciones para satisfacerla es que, por parte de varias Universidades latinoamericanas se han creado carreras llamadas Física Tecnológica (Argentina), Ingeniería Física, (Colombia, Cuba, Chile, México y Perú) Instrumentación o simplemente Física Aplicada (Brasil). A modo de ejemplo, algunas de las instituciones de los países mencionados que han decidido satisfacer esta nueva necesidad de carreras en el ámbito de la física aplicada son: Centro Atómico Bariloche (Argentina), Univ. de Sao Paulo (Brasil) y Univ. Estatal de Campinas (Brasil), Univ. Nacional de Colombia (Colombia), Univ. EAFIT (Colombia), Univ. Andrés Bello (Chile), Univ. de Santiago de Chile (Chile), Univ. de Concepción (Chile), Univ. Nacional Autónoma de México (México), Univ. Autónoma de Yucatán (México) y Pontificia Univ. Católica del Perú (Perú). En todos los países citados, los argumentos esgrimidos para la creación de estas nuevas carreras son similares. Aunque en cada caso tienen grados de desarrollo muy diferentes y responden a necesidades concretas de cada uno de éstos.

Específicamente en Chile, sin pretender hacer un análisis comparativo entre los diversos planes de estudio ofrecidos por las diferentes Universidades nacionales, no podemos dejar de mencionar que se han creado tres carreras universitarias con el mencionado perfil, éstas son ofrecidas por la Univ. Andrés Bello, Univ. de Concepción y Univ. de Santiago. Si bien, todas estas nuevas carreras responden a inquietudes muy parecidas, en cada plantel se le imprimieron diferentes matices, dependiendo de la visión y de las fortalezas de cada una de las instituciones educacionales 
involucradas. A nivel internacional, el Instituto Tecnológico de California y la Universidad Cornell en Estados Unidos tienen un reconocido prestigio.

Las tres universidades incluidas en la Tabla 1 otorgan el título de Ingeniero Físico y la duración de la carrera en todas ellas es de seis años. Un objetivo específico de este trabajo es mostrar algunas de las motivaciones más relevantes para la creación de la especialidad de Ingeniería Física de la Universidad de Concepción. Además, describiremos esta especialidad desde su origen hasta las actuales estrategias para consolidar dicha especialidad. En la Tabla 1 se describen algunos detalles de las carreras de Ingeniería Física.

Tabla 1: Carreras de Ingeniería Física impartidas en algunas Universidades Chilenas

\begin{tabular}{|l|c|c|}
\hline Institución & Año de creación & Referencia \\
\hline Univ. Andrés Bello & 2006 & www.unab.cl \\
\hline Univ. de Concepción & 2003 & www.udec.cl \\
\hline Univ. de Santiago & 1994 & www.usach.cl \\
\hline
\end{tabular}

\section{DESARROLLO DE LA ESPECIALIDAD}

Analizando las posibilidades que se habían generado para los profesionales que, con una fuerte formación en ciencias físicas, tenían el deseo de dedicarse por completo a la aplicación de dicha ciencia, concentrando todos sus esfuerzos en desarrollar nuevas tecnologías o adaptar tecnologías existentes a situaciones específicas, para así lograr optimizar procesos productivos, idea ya desarrollada parcialmente en el trabajo de Jackson, (1972).

A comienzos de los años 2000, un grupo de académicos del Departamento de Física de la Universidad de Concepción, se abocaron al estudio para la creación de una carrera que satisficiese esta creciente necesidad. Dicho grupo de científicos, notó que la formación tradicional de las licenciaturas en física no era suficiente, ya que estos profesionales carecían de algunas herramientas básicas como lo son la evaluación y optimización de proyectos, desarrollo de prototipos y experiencia laboral mediante prácticas profesionales en la industria durante los periodos de estudios y de algunos aspectos más técnicos como capacitación en electrónica, procesamiento de información, etc. En el año 2003, es creada la especialidad de Ingeniería Física en la Universidad de Concepción (Decreto UDEC, 2005) para contribuir a fortalecer la competitividad industrial por medio de la innovación, tanto regional como nacional, ingresando los alumnos a partir del año 2004.

\section{Aspectos Relevantes de la Malla Curricular}

El Ingeniero Físico de la Univ. de Concepción es un profesional que, gracias a una sólida formación orientada hacia las aplicaciones de las Ciencias Físicas, estará preparado para resolver problemas y aportar con soluciones en un amplio espectro en Investigación y Desarrollo, hábil en comprender los problemas de la transferencia tecnológica y competente para comunicar efectivamente los complejos temas sobre la ciencia y la tecnología a las futuras generaciones. Actualmente la especialidad cuenta con más de 30 estudiantes, esperando una tasa de ingreso de alrededor de 10 alumnos por año. El nivel de los estudiantes que han ingresado los últimos años al plan común de la carrera (Física e Ingeniería Física) ha mejorado notablemente, como se muestra en la figura 1. En la actualidad los estudiantes más avanzados se encuentran finalizando su tesis de titulación.

El Ingeniero Físico estará entrenado para enfrentar un problema real, descomponerlo en sus constituyentes básicos, traducir cada parte en expresiones matemáticas, manipular esas 
expresiones para obtener nuevos resultados y entonces trasladar esos resultados matemáticos de regreso a sentencias de la realidad física. Esta habilidad es importante y común en un amplio campo de actividades donde se requiere entender el comportamiento de sistemas y mecanismos con el propósito de realizar predicciones y ganar control sobre ellos.

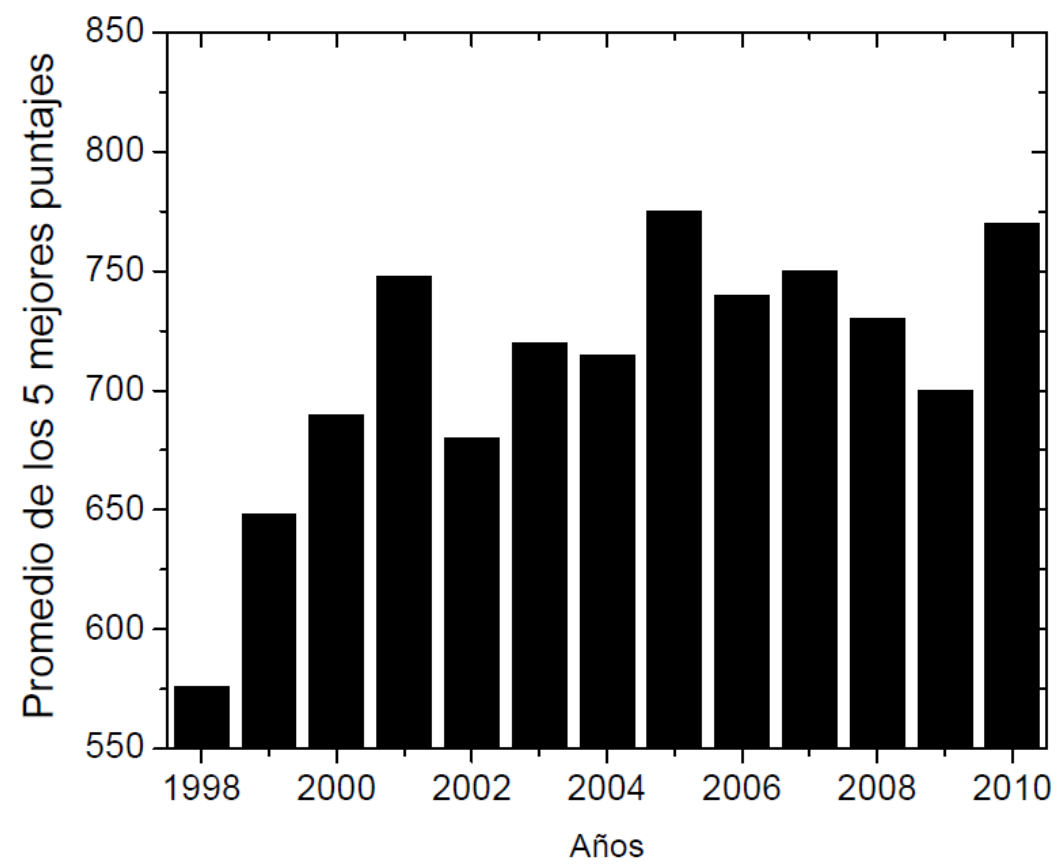

Fig. 1: Promedio de los cinco más altos puntajes de ingreso en los últimos 13 años a la carrera de Ciencias Físicas impartida en la Universidad de Concepción.

Para lograr el perfil mencionado en los párrafos anteriores, se cuenta con los recursos humanos, bibliográficos, infraestructura y los siguientes laboratorios de investigación fundamental y aplicada:

a.- En el Departamento de Física de la Univ. de Concepción se dispone de los siguientes laboratorios: teledetección satelital e instrumentación óptica, óptica cuántica, pinzas ópticas, fenómenos fototérmicos y fotoacústica, fluorescencia de rayos-X por reflexión total (TXRF).

b.- Además, en las diferentes facultades de la Univ. de Concepción nuestros alumnos cuentan con amplias facilidades para la utilización de más de 50 laboratorios en el área de ciencias aplicadas.

La malla curricular (ver Tabla 2) de ésta especialidad ha sido estructurada de la siguiente forma:

i) Plan común: Los estudiantes ingresan a un plan común, que tiene una duración de cinco semestres y en ellos el estudiante adquiere una formación teórica y experimental. A partir del sexto semestre, el estudiante elige la especialidad que desea seguir (física o ingeniería física). En este plan se introducen los conceptos fundamentales de la física clásica. Aquí el estudiante aprende los conceptos, principios y leyes fundamentales de la física. Entre otros, se tienen cursos de álgebra, cálculo, ecuaciones diferenciales, física computacional, electromagnetismo, mecánica clásica, óptica, etc. Se complementan las clases teóricas con el trabajo experimental guiado y semiguiado. En este periodo se inicia el estudio riguroso de la descripción de fenómenos naturales, en asignaturas tales como: electrodinámica I, mecánica de fluidos, mecánica cuántica I y física estadística I.

ii) Especialidad: Tiene una duración de siete semestres. A partir del sexto semestre, el alumno debe cursar siete asignaturas obligatorias de especialidad como por ejemplo: dispositivos semiconductores, electrónica, circuitos digitales, procesamiento de señales e imágenes, métodos matemáticos y estadísticos de la física, diseño de prototipos I y II etc. más siete asignaturas electivas y dos asignaturas de tópicos en física, que son de carácter libre y el alumno se hace partícipe de su propia formación. En las asignaturas de tópicos el alumno propone un programa 
semestral de actividades que le permita profundizar en un tema de su interés particular. Además, en este periodo se continúa incentivando a los estudiantes a la realización de pasantías en la empresa. Finalmente, deben cursar una asignatura de proyecto de tesis y dos asignaturas de Tesis. En Tabla 2 se muestran todas las asignaturas de la carrera.

Tabla 2: Malla curricular de la carrera Ingeniería Física

\begin{tabular}{|l|l|}
\hline I Semestre & VII Semestre \\
Algebra y Trigonometría & Mecánica de Fluidos \\
Física I & Mecánica Cuántica I \\
Computación Científica & $\begin{array}{l}\text { Electrónica } \\
\text { Electivo I } \\
\text { Física Computacional III }\end{array}$ \\
\hline II Semestre & VIII Semestre \\
Cálculo Diferencial e Integral & Física Estadística I \\
Algebra Lineal & Física Estado Sólido \\
Física II: Fundamentos de Mecánica & Circuitos Digitales \\
& Diseño de Prototipos I \\
& Electivo II \\
\hline III Semestre & IX Semestre \\
Ecuación Diferencial Ordinarias & Electivo III \\
Cálculo III & Electivo IV \\
Física Matemática I & Electivo V \\
Física III -1: Electromagnetismo I & Tópicos en Física I \\
& Diseño de Prototipos II \\
\hline IV Semestre & X Semestre \\
Física Computacional II & Procesamiento de Señales e \\
Cálculo IV: Cálculo Complejo & Imágenes \\
Física III-2: Electromagnetismo 2 & Electivo VI \\
Física IV: Termodinámica & Electivo VII \\
Laboratorio I & Tópicos en Física II \\
\hline V Semestre & Proyecto de Tesis \\
Física Matemática II & XI Semestre \\
Física V: Óptica & Tesis I \\
Mecánica Clásica I & \\
Física VI: Oscilaciones y Ondas & \\
Teoría de Circuitos & \\
\hline VI Semestre & \\
Física Matemática III & \\
Física VII: Intr. Mecánica Cuántica & \\
Electrodinámica I & \\
Dispositivos Semiconductores & Tesis II \\
Laboratorio II & \\
\hline
\end{tabular}

Una característica importante de nuestra carrera es que, desde muy temprano se inicia a nuestros alumnos en la lectura de artículos científicos y desarrollo de experimentos fundamentales. En el ciclo de especialidad, deben sumirse en el área de física aplicada por medio del desarrollo de diseño de prototipos y prácticas profesionales, para dar soluciones concretas a requerimientos de la industria. El plan de estudios de nuestra carrera es suficientemente flexible, como para permitir al estudiante elegir asignaturas que le sirvan a futuro en el área donde quiera desarrollarse 
profesionalmente. Como resultado de este proceso formativo se espera que el estudiante sea capaz de formular un determinado problema en lenguaje científico, de discernir acerca de la factibilidad de resolverlo por medio de determinados procedimientos y finalmente implementar una solución. A la fecha hay pocos alumnos egresados y por lo tanto pocos los estudiantes que han realizado prácticas profesionales. Sin embargo, la opinión que tienen los empresarios es muy positiva, lo que permite augurar un buen desempeño de los futuros profesionales.

El grado de Licenciado en Ciencias Físicas se obtiene una vez aprobado el cuarto año. Adicionalmente el estudiante debe cursar un periodo de dos años, completando así un total de seis años de estudios formales, para obtener el título profesional de ingeniero físico.

\section{Requisitos de titulación para Ingeniería Física}

Para ser acreedor al título profesional de Ingeniero Físico se debe haber aprobado un mínimo de 231 créditos, la totalidad de las asignaturas (obligatorias, electiva y de especialidad) contempladas en el plan de estudios de la carrera (en total doce semestres), y haber realizado y defendido exitosamente la tesis.

\section{CONCLUSIONES}

A partir del análisis de la propuesta de la carrera de Ingeniería Física se pueden extraer las siguientes conclusiones:

i) La propuesta y creación de esta nueva especialidad responde a la necesidad de innovación del sistema productivo y no simplemente a un requerimiento académico.

ii) El sistema productivo ha abierto las posibilidades para que los estudiantes de esta especialidad se inserten y puedan contribuir en dicho sistema, esto se hace evidente por la buena acogida que han tenido nuestros alumnos en sus prácticas profesionales.

iii) El aumento de los puntajes de ingreso a la carrera, deja de manifiesto el interés de los estudiantes por esta nueva especialidad y la mejora en la calidad de éstos.

iv) Aunque el número de estudiantes titulados es escaso, su desarrollo profesional ha sido claramente exitoso. De mantenerse las tendencias actuales, en lo que se refiere a los puntajes de ingreso, implicaría que los alumnos ingresan con un mejor nivel académico facilitando su formación profesional. Por lo expuesto es esperable que nuestros próximos profesionales deberían estar mejor capacitados para ser un real aporte al sistema productivo por medio de la innovación y creación de nuevos productos y servicios, respondiendo así a necesidades del mercado.

\section{AGRADECIMIENTOS}

Los autores agradecen a la facultad de Ciencias Físicas y Matemáticas y al Departamento de Física de la Universidad de Concepción-Chile por el apoyo brindado para la realización de este trabajo.

\section{REFERENCIAS}

Castrillón, M.J. y otros dos autores; La ingeniería física y las ciencias biomédicas. ¿Una simbiosis con futuro? Revista facultad ciencias de la salud, Universidad del Cauca, Colombia, 11(2), 19-26 (2009).

CEPAL; Vínculo entre universidades y sector productivo es clave para lograr sociedades más inclusivas (2010) (en línea). http://www.revistasumma.com/economia/7512-cepal-vinculo-entreuniversidades-y-sector-productivo-es-clave-para-lograr-sociedades-mas-inclusivas.html Acceso: Abril de (2011). 
Decreto UDEC, Modificación de Plan de Estudios. Carrera de Ciencias Físicas y Astronómicas, Fac. de Ciencias Físicas y Matemáticas, Decreto № 2005-051. Univ. de Concepción (2005).

Flores, J.A y otros dos autores; Ingeniería física. Una nueva perspectiva de la enseñanza de la física. Revista de la educación superior, 10(40), 1-11 (1981).

Jakcson, G. Physics and Engineering, Phys. Educ. 7, 74-77 (1972).

López, N. y otros dos autores; Generación y aplicación de conocimiento por la empresa industrial: factores determinantes. Decisiones basadas en el conocimiento y en el papel social de la empresa: XX Congreso anual de AEDEM, (2007). http://dialnet.unirioja.es/servlet/articulo?codigo=2487698. Acceso: Diciembre de (2010).

Moya-Angeler, J. (Ed.), Innovación y desarrollo económico, Colección Mediterráneo Económico, Vol. 17, 11-28, Fundación Cajamar, España, (2010).

OMPI-CEPAL; Reunión Regional OMPI-CEPAL de Expertos Sobre el Sistema Nacional de Innovación: Propiedad Intelectual, Universidad y Empresa, Santiago-Chile, 1 y 3 de Oct. 2003 http://www.wipo.int/edocs/mdocs/sme/es/ompi_cepal_inn_san_03/ompi_cepal_inn_san_03_t1_2.pdf Acceso: Abril de (2011).

UDEC; Ingeniería Física, Departamento de Física, Facultad de Ciencias Físicas y Matemáticas, Universidad de Concepción, Concepción, Chile. www.udec.cl/carreras. Marzo (2011).

UNAB; Ingeniería Física, Universidad Andrés Bello, Santiago, Chile.

http://www.unab.cl/admision/carreras/ficha/ingenieria-fisica/santiago/diurno/default.aspx. Marzo (2011).

USACH; Ingeniería en Física, Departamento de Física, Facultad de Ciencias, Universidad de Santiago, Santiago, Chile. http://fisica.usach.cl/ ingfisica. Marzo (2011).

Yates, W.R; Lehigh University: A History of Education in Engineering, Business, and the Human Condition. Bethlehem [Pa.], Lehigh University Press; London; Cranbury, NJ: Associated University Presses.. 334. (1992). 
